\title{
A literature Review: Do Rapid Response Systems reduce the incidence of major adverse events in the deteriorating ward patient?
}

\section{Objectives}

To conduct a literature review that explores the impact of Rapid Response Systems on reducing major adverse events experienced by deteriorating ward patients.

\section{Background}

Patients located on hospitals wards are older, often have multiple co-morbidities and are often at risk of life threatening clinical deterioration. Rapid Response systems have been developed and implemented to provide appropriate and timely intervention to these patients.

\section{Design}

A comprehensive review of the literature.

\section{Methods}

This review used the Rapid Response System framework recently developed by experts in the area of Rapid Response System. MEDLINE, CINAHL, EMBASE and Cochrane databases were searched from January 1995 to June 2009. 16 papers were selected that most clearly reflected the research aim. Each paper was critically appraised and systematically assessed. Major themes and findings were identified for each of the studies.

\section{Results}

The effectiveness of Rapid Response Systems in reducing major adverse events in the deteriorating ward patients remains inconclusive. Six Studies demonstrated that the introduction of a Rapid Response System positively impacted on patient outcomes but three studies demonstrated no positive impact on patient outcomes. Nursing staff appear reluctant to utilise Rapid Response Systems but the rationale for this is unclear. However, the continued underutilisation and inactivation may be one reason why research findings evaluating Rapid Response Systems have been inconclusive.

\section{Relevance to clinical practice}


Rapid response systems are multidimensional models. They are relatively new innovations that have important implications for clinical research and implementation policy. This review contributes to the emerging debate on Rapid Response Systems.

\section{Conclusions}

The paper illustrates two important gaps within the literature. Firstly, 'ramp up' systems have not been subjected to formal evaluation. Secondly, Rapid Response Systems are under activated and underutilised by nursing staff. There is an urgent need to explore the reasons for this and identify interventions to improve the activation of these systems in an effort to promote safe and effective care to the deteriorating ward patient.

Key words: Rapid Response Systems, the deteriorating patient, integrative review, Medical Emergency Teams, Critical Care Outreach Teams. 


\section{Introduction}

The increasing demand for hospital beds, coupled with an ageing population and shorter hospital stays have resulted in increased patient acuity (Australian Institute of Health and Welfare, 2008). These patients are at risk of clinical deterioration, which can have life threatening consequences (McGloin et al., 1999 Buist et al., 2003; Massey et al., 2008). These consequences including in-hospital cardiac arrest, unplanned admission to the intensive care unit (ICU) and death have been termed major adverse events (MAEs) (Buist et al., 1999; Buist et al., 2004; Hillman et al., 2005; Cretikos et al., 2006;).

Rapid Response Systems (RRS) have been developed to improve the morbidity and mortality in acutely ill ward patients who experience acute physiological deterioration (Buist et al., 2002; Cretikos \& Hillman, 2003; Bellomo et al., 2004; DeVita et al., 2006; Cretikos et al., 2007a). These systems have been developed in Australia (Hillman et al., 2005), USA (DeVita et al., 2006) and Europe (Priestley et al., 2004). RRS are hospital wide systems that provide a safety net for ward patients who suddenly deteriorate and develop complex care needs that may be outside the scope of clinical ward staff knowledge and skills (DeVita et al., 2006). RRS have an afferent (case detection and response-triggering) limb and an efferent (response) limb to successfully prevent deterioration or to respond appropriately to the deteriorating patient (figure 1.0) (DeVita et al., 2006). RRS usually consists of an medical officer and a nurse (DeVita et al., 2006) who assesses the patient and institutes emergency therapy to stabilise the patient's clinical condition. Previous systematic reviews of RRS have evaluated only the afferent limb (Gao et al., 2007) or the efferent limb (Esmonde et al., 2006; McGaughey et al., 2007) thus, preventing a comprehensive evaluation.

This literature review examined the research on the effects of RRS on reducing inhospital cardiac arrests, unplanned admission to ICU and patient mortality. The review aims to improve knowledge and understanding of these systems and through this analysis identify strategies for promoting the optimal use of these systems. 


\section{Methods}

Well-defined literature search strategies are critical for enhancing the rigor of the review (Whittemore \& Knafl, 2005). To identify appropriate literature on the topic, the following databases were searched: CINAHL, MEDLINE, EMBASE and Cochrane. Search terms that were used included Medical Emergency Teams (MET), Rapid Response Systems (RRS), Rapid Response Team (RRT), Critical Care Outreach (CCO), Intensive Care Liaison Nurse, Early Warning Scores (EWS) and Modified Early Warning Scores (MEWS), critically ill ward patients, acutely ill ward patients, and deteriorating ward patients. The search was restricted to the English Language and by patient age category (all adults over 16 years). Although computerised databases are efficient and effective, limitations associated with inconsistent search terms may only yield about $50 \%$ of eligible studies (Whittemore \& Knafl, 2005). Therefore ancestry searching and journal hand searching were also used to ensure all relevant literature was accessed (Conn \& Rantz, 2003).

\section{Inclusion/exclusion criteria}

Literature was included if it was published between 1995-2008. The contemporary nature of the topic dictated the chosen timeframe. The concept of the deteriorating ward patient and RRS were only just being recognised and studied 13 years ago. Studies were included if they provided empirical data on any of the three key outcomes of inhospitable cardiac arrest, unplanned admission to ICU and hospital mortality and if they described interventions initiated by the RRS. Studies were excluded if they did not use primary data collection or if they evaluated other aspects of the RRS, for example, team performance or the introduction of a RRS.

Issues related to data collection, data extraction, analysis and synthesis need to be explicit and transparent otherwise a literature review may be criticised as being subjective and inaccurate (O'Mathuna, 2000). This review demonstrates this transparency through a clear description of the analysis and findings and the use of a theoretical framework outlined by Whittemore and Knafl (2005). This framework involves the following stages:

$>$ Problem identification

$>$ Literature search

$>$ Data analysis

$>$ Discussion 


\section{Problem identification}

The initial stage of any review method requires a clear identification of the problem. A well-specified research purpose and question facilitates the extraction of appropriate data from the identified primary sources (Whittemore \& Knafl, 2005). Evaluation of RRS effectiveness and efficacy is problematic for a number of reasons. Firstly, a wide variation in terminology exists (Ball et al., 2003; Bellomo et al., 2003; Buist et al., 2004). Terms such as Medical Emergency Teams, Rapid Response Teams and Critical Care Outreach Teams are often used interchangeably and may only describe one limb of the system. Secondly, many of the structures and processes of the system are fundamentally different for example, the times the service is available to patients and staff (DeVita et al., 2006; Robson, 2002). In an attempt to address these issues, a recent consensus statement written by experts produced clear recommendations that clarify the nomenclature in this area (DeVita et al., 2006). One of the key messages from this consensus statement is that RRS incorporate two separate but interlinked limbs: the afferent limb and the efferent limb (figure 1). The complexities of the different RRS and the contemporary nature of the model proposed by DeVita et al (2006) means that the application of this model to clinical practice has yet to be clearly demonstrated. This paper explored two essential elements of the model; the efferent and the afferent limb.

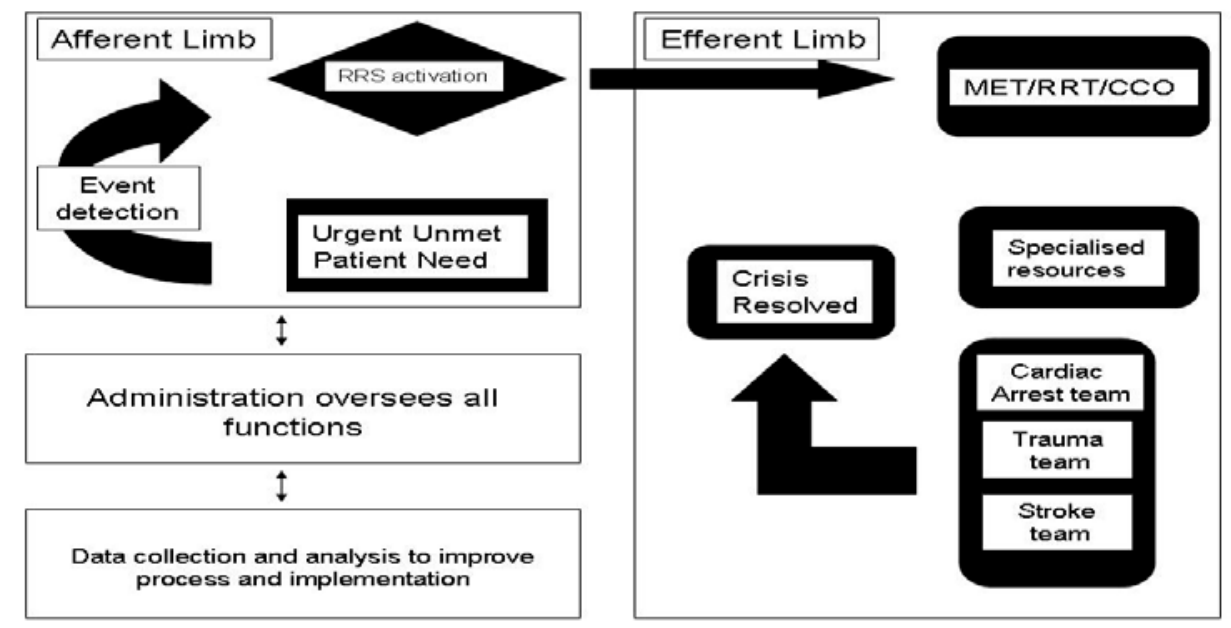

Figure 1.0 Rapid Response System

Key to terms; MET (Medical Emergency Team), RRT (Rapid Response Team), CCO (Critical Care Outreach). 
In summary, this section of the review has identified a number of problems that require addressing. First, there is lack of data examining the effect of both limbs of the RRS on serious adverse events in the deteriorating ward patient. Second, the model proposed by DeVita and colleagues (2006) has not yet been used in research. This paper will use the efferent and afferent limb of the model to present the findings thereby providing a comprehensive and inclusive overview of the topic. The use of this model in this form will also enable examination of the models relevance and effectiveness to clinical practice.

\section{Data Evaluation}

Once the literature was accessed, data were extracted for analysis and synthesis. Each paper was critically appraised and systematically assessed by all the authors for the following characteristics: purpose statement or research questions, study design, sample size, characteristics of subjects, method of data analysis, and results. Major themes and findings were identified for each of the studies. This process was essential to ensure methodological rigor (Brown et al., 2003). The quality of included studies was determined by evaluating their internal validity, "the extent to which the study design, conduct, and analysis systematically avoided or minimised potential sources of bias” (Conn \& Rantz, 2003). A total of 581 papers were initially identified. After the inclusion and exclusion criteria were applied 16 papers were included in the review.

\section{Data Analysis}

The next stage in the review process is data analysis (Whittemore \& Knafl, 2005). Data from the included studies were ordered, categorised and summarised to produce a coherent and logical summary of the data. The next section of this review presents the data analysis stage of the review. Each limb of the RRS is presented, discussed and analysed separately to capture the complexities of the issues.

\section{The Afferent limb: Track and/or trigger systems}

A necessary function of any RRS is a clear method of detecting "emergent unmet patient needs" (DeVita et al., 2006). The afferent limb is responsible for detecting patients at risk of clinical deterioration and triggering a response to that deterioration. 
Most RRS use a set of predetermined objective criteria that involve the assessment, measurement and documentation of patients' vital signs (DeVita et al., 2006; Cretikos et al., 2007a; Cuthbertson et al., 2007). These criteria are increasingly referred to as track and trigger systems (TTS) (Gao et al., 2007), and they represent an afferent limb intervention. The aim of the TTS is to ensure timely recognition of all patients at risk of acute deterioration and to ensure attendance by appropriately skilled staff.

Currently two main types of TTS exist (Gao et al., 2007). The single parameter system uses periodic observations of selected vital signs and then compares these vital signs to a set of criteria with a predefined threshold; a response algorithm is activated when any criterion is met. (Cretikos et al., 2007b; Gao et al., 2007). The aggregate weighted scoring system allocates a weight to predefined physiological criterion as a function of its abnormality and a summary score is derived (Subbe et al., 2003; Cuthbertson et al., 2007; Gardner-Thorpe et al., 2006). One of the most well know aggregate track and trigger system is the Early Warning Score (EWS) (Morgan \& Wright, 1997) this was based on five physiological parameters: systolic blood pressure, pulse rate, respiratory rate, temperature and the mnemonic AVPU (alert, voice, pain, unresponsive). Many of the aggregative scoring systems currently in use have been adapted and modified from Morgan \& Wrights' (1997) original EWS, however the EWS has only been published in an abstract form and as such it is difficult to assess its sensitivity and specificity in responding to patients at risk of clinical deterioration. Because of the different TTS in clinical use there is a lack of consensus as to the most effective, sensitive and valid tool (McArthur-Rouse, 2001; Esmonde et al., 2006; Gao et al., 2007). Despite, this lack of agreement, the National Confidential Enquiry into Patient Outcome and Death (NCEPOD) (Cullinane et al., 2005) and DeVita and colleagues (2006) recommend the use of some form of TTS by health-care providers. More recently the National Institute for Clinical Effectiveness (NICE) has also recommended the use of TTS to improve the safety and outcomes of the acute ward patient at risk of clinical deterioration (NICE, 2007).

The sensitivity and specificity of aggregate systems have been reported in five papers (Table 1) (Stenhouse et al., 2000; Subbe et al., 2003; Subbe et al., 2001; Needleman et al., 2002; Gardner-Thorpe et al., 2006). The Modified Early Warning Score (MEWS) is an example of afferent intervention and the use of the score leads to earlier 
identification of surgical patients at risk of a MAE (Stenhouse et al., 2000). The impact of using the MEWS on surgical patients has also been evaluated by Gardner and colleagues (2006). Fifty seven (17\%) of the 334 ward patients triggered the activation algorithm. Sixteen (5\%) of the total were admitted to ICU or the high dependency unit (HDU). The sensitivity and specificity of the MEWS in this study using the score of 5 or more was $75 \%$ and $83 \%$ respectively for ICU or HDU admission. However the study only included patients who had undergone colorectal surgery and as such it possible the findings may not apply to the wider hospital population. Post-operative patients may be more sensitive to physiological changes than other patient groups and may therefore exhibit more obvious signs and symptoms of clinical deterioration. Subbe et al., (2001) also investigated the ability of the MEWS to identify medical patients at risk of clinical deterioration. The study used a MEWS of 5 and demonstrated that a MEWS score of 5 or more was associated with an increased risk of mortality in medical patients.

In an attempt to develop robust and sensitive aggregate scoring systems and therefore more sensitive afferent interventions risk factors associated with increased mortality have been examined in two studies (Table 1). Hodgetts et al., (2002) identified significant predicators of cardiac arrest as dyspnoea, abnormal respiratory rate, abnormal pulse, reduced systolic blood pressure, abnormal temperature, reduced pulse oximetry and nurse or doctor concern. This was the first evidenced-based approach to provide activation criteria for a RRT. An observational population-based single centre study also investigated the contributions that vital signs had on morbidity (Duckitt et al., 2007). Analysis of the data from this study demonstrated that a respiratory rate $>$ 20/minute, heart rate, >102/minute, systolic BP < $99 \mathrm{mmHg}$, temperature <35.3 C, oxygen saturation $<96 \%$ and disturbed conscious levels were associated with increased mortality. Using this information, Duckitt et al., (2007) then developed a simple early warning scoring tool that has since been validated by the researchers (Duckitt et al., 2007).

The single parameter system has been evaluated using a nested matched case control study to assess the ability of the MET activation criteria to identify patients at risk of a MAE (Cretikos et al 2007). This study like the previous studies (Hodgetts et al., 2002 Duckitt et al., 2007;) confirmed that in combination, increased heart rate, respiratory rate, low systolic blood pressure and a decrease in the Glasgow Coma Score (GCS) 
were specific predictors of cardiac arrest, unplanned ICU admission and unexpected death.

\section{The Efferent limb of a Rapid Response System}

The two most common types of response teams are the "high-capability team" and the “ramp-up team” (DeVita et al., 2006). The MET and the Rapid Response Team (RRT) are examples of "high-capability teams" whilst Critical Care Outreach (CCORT) team and or the ICU liaison nurse are examples of "ramp-up teams" Although each of these individual efferent teams have been investigated in a number of different clinical settings, no studies exist which demonstrate outcome differences between the different efferent teams.

CCOTs have been developed predominantly in the UK and are perhaps the most well know of the "ramp-up" teams. They implement interventions through the use of protocols, standing orders and patient group directives (McArthur-Rouse, 2001; Coombs \& Dillon, 2002; DeVita et al., 2006) and are usually nurse led teams, thus differing from the "high-capability" team which are physicians led. CCOT vary significantly between different hospitals (Robson, 2002) for example, they may be resourced by a single critical care nurse or a team of critical care nurses, hours of operation also differ between different hospitals with them operating during normal office hours, or extending the service to weekends, or 24 hours. The differences in the organisation, structures and processes of these teams make a comprehensive assessment of their effectiveness difficult.

The value and role of CCOT in improving clinical outcomes in the deteriorating ward patient remains difficult to quantify. Two systematic reviews recently undertaken on CCOTs found that the lack of high quality studies prevented clear recommendations being developed on the role of CCOT in improving acutely ill ward patients clinical outcomes (Esmonde et al., 2006; McGaughey et al., 2007)). However, McGaughey’s paper only included two studies (Priestley et al., 2004; Hillman et al., 2005) and one of the included studies was a prospective cluster randomised trial evaluating the effectiveness of the MET (Hillman et al., 2005). The MET is an example of a highcapability team and therefore it is questionable if this study should have been included in this review. Esmonde at al., (2006) undertook a systematic review of CCOTs and, 
concluded that although improvements in patient outcomes were linked to the CCOT the differences in service delivery and the methodological diversity of the studies made it difficult to produce clear recommendations on the value and role of CCOT in reducing the incidence of adverse events. Like McGaughey et al., (2007), this systematic review also included studies evaluating the effectiveness of the MET and given the differences between the two systems it remains problematic to generalise the findings of this paper to the wider acute ward patient population.

Despite the lack of clear recommendations in relation to the role of CCOT on improving deteriorating ward patients clinical outcomes, (Esmonde et al., 2006; McGaughey et al., 2007) a randomised control trail evaluating the effectiveness of the CCORT study demonstrated a statistically significant reduction in hospital mortality in wards where CCOTs operated compared to wards where it did not operate (Priestley et al., 2004). This step-wise ward randomised trial with a phased introduction of CCOT intervention was conducted in 16 acute adult wards of one general hospital in the United Kingdom over a 32-week period. A sequenced introduction of the intervention may have led to a contamination of the study wards. That is the control wards may have been exposed to CCOT or MET like activities for example, earlier assessment and intervention.

In summary ramp-up teams have been implemented with the aim of improving the clinical outcomes of the deteriorating ward patient and the management processes involved in caring for these vulnerable patients. These teams have been operational for a number of years however there is paucity of high quality data validating the use of teams in reducing in-hospital cardiac arrest, unplanned admission to ICU and death.

High-capability team; are usually physician led and available 24 hours a day, seven days a week (Lee et al., 1995; Buist et al., 2002; Cretikos \& Hillman, 2003;). Two systematic reviews have evaluated the effectiveness of high-capability teams (Ranji et al., 2007; Winters et al., 2007). Both reviews concluded that the evidence available on the effectiveness of high capability teams was inconclusive. Further they both argue that that many of the positive improvements in patient outcomes were the result of improvement in the care and management of deteriorating ward patients and technological developments. In their conclusion and recommendations these two reviews call for more large randomised trials to clarify the efficacy of RRS. 
Five single centre non-randomised studies (Table 2) have demonstrated that the MET was effective in reducing in-hospital cardiac arrests, hospital mortality and unplanned admission to ICUs (Buist et al., 2002; Bellomo et al., 2003; DeVita et al., 2004; Jones et al., 2005; Dacey et al., 2007). In a non-randomised before and after cohort study Buist et al., (2002) demonstrated a 50\% reduction in hospital cardiac arrests. In a single centre cohort study using historical controls, (DeVita et al., 2004) demonstrated, a 17\% reduction in the incidence of in-hospital cardiac arrests. Bellomo et al., (2003) also reported a significant reduction in the number of in-hospital cardiac arrests and hospital mortality following the implementation of a MET. This cohort study also used historical controls. Jones et al. (2005) reported a sustained and progressive reduction in cardiac arrests in their cohort study using historical controls but, this study took four years to complete. The most recent study evaluating the impact of a high capability RRS on in-hospital cardiac arrests, unplanned admission to ICU and hospital mortality was conducted by Dacey et al., (2007). The results of this study also demonstrated a significant reduction in hospital cardiac arrests and unplanned admission to ICU.

The majority of studies demonstrating the positive impact of the MET on patient outcomes have been undertaken in Australia. Australia has a different health care system to other Western Countries making generalisations of these finding to other health care systems problematic.

Despite promising results that high capability team's impact positively on patient outcomes there is a growing body of literature demonstrating unconvincing evidence regarding the effectiveness of these teams on patient outcomes. Three studies (Table 2) have failed to demonstrate that the MET positively influences hospital cardiac arrests, unplanned admission to ICU and hospital mortality (Bristow et al., 2000; Bellomo et al., 2004; Hillman et al., 2005; Kenward et al., 2004). Kenward et al. (2004) evaluated the impact of a MET one-year after implementation. This single centre cohort study used historical controls and failed to reveal any statistical significance of a MET intervention on in-hospital cardiac arrests and hospital mortality. Similar to the Hillman et al., (2005) study, this study also revealed low activation of the MET even when activation criteria were present. The only randomised control trial conducted on the MET failed to reveal any differences between the intervention and the control hospitals in the primary outcome measures of in-hospital cardiac arrest, hospital mortality and 
unplanned admission to ICU (Hillman et al., 2005). Although the findings of this study imply that the MET is ineffective in improving the three outcome measures, there are a number of possible explanations for these findings. First, the timeframe for the implementation of the MET study may have been too short, there was a low documentation of vital signs which may have impacted on the number and timing of MET activation and finally there was a low activation of the MET even when activation criteria were present. Bristow et al., (2000) evaluated the effectiveness of the MET in reducing the rates of in-hospital cardiac arrest, unplanned admission to ICU and hospital mortality. This non-randomised cohort study with casemix adjustment found no significant difference in the rates of cardiac arrest and hospital mortality between the intervention hospital and the two control hospitals. This study, like other studies (Hillman et al., 2005; Cretikos et al., 2007b), argues that patients exhibited premonitory signs of an impending adverse event that should have activated a MET response. However out of 706 MET that should have been activated only 150 were actually called. This clearly highlights that the MET is underutilised and not activated appropriately, and this could have impacted significantly on the findings of this study.

\section{Discussion}

\section{The Afferent limb: Track and Trigger Systems}

The evidence from this review highlights that many hospitals have developed and implemented their own TTS, yet these tools have not been subjected to rigorous testing or evaluation. Several limitations to the use of the MEWS to predict patient deterioration exist. First, response times for the MEWS are not standardised, which may lead to delayed responses, inadequate assessment and inadequate treatment interventions and patient management. Second, a score from a single parameter does not always translate into an increased risk of clinical deterioration. For example, abnormal respiratory rate or documented shortness of breath is significantly associated with cardiac arrest compared to changes in urine output (Fieselmann et al., 1993 Cretikos et al., 2008). It may therefore be appropriate when using aggregate scoring systems to assign a higher weighting to different parameters however further research would be required to determine which parameters this maybe applicable to. The literature seems to suggest that many aggregate scoring systems continue to use physiological variables that have demonstrated limited predictive value (Kellett \& Deane, 2006; Cuthbertson et al., 2007). Threshold values also differ between different 
systems (Esmonde et al., 2006; Gao et al., 2007; Cretikos et al., 2008) and different studies (Goldhill \& McNarry, 2004; Cuthbertson et al., 2007; Gao et al., 2007;), which can make generalisations problematic. Threshold scores for triggering an intervention should be derived from good quality empirical data. Therefore this review suggests that the role and importance of individual physiological variables require further study. In spite of these limitations it must be acknowledged that the role of any early warning score is to secure timely and appropriate intervention. These TTS have not been developed as predictors of outcomes, however given that the afferent limb of the RRS has been identified as an important element of the system, it is essential that this aspect of the system is able to demonstrate its contribution to the overall objectives of the RRS.

\section{The Efferent limb of the RRS}

Currently, there are two similar yet distinct efferent models of the RRS, CCOTs, and ICU liaison nurses referred to as a "ramp-up system,” and the RRT (Rapid Response Team), referred to as a "high-capability system'. Evidence regarding the effectiveness of the efferent limb of RRS remains inconclusive and the only randomised controlled trial that has been conducted on RRS (Hillman et al., 2005) failed to significantly demonstrate a reduction in major adverse events. However, despite these inconclusive findings RRS have achieved widespread adoption in the acute care sector. Additionally, the Institute of Healthcare Improvement (IHI) in the USA actually recommend the implementation of RRS in acute hospitals and the Australian Commission on Safety and Quality in Healthcare (2008) also recommend RRS be implemented to suit local needs. In the United Kingdom the National Institute for Clinical Excellence, which bases its recommendations on systematic reviews of the best available evidence, recommends the introduction of RRS in acute care settings (NICE 2007).

RRS are clearly highly complex systems and many different variables impact on their effectiveness. Even when the most robust methodology is used, it may still not be possible to quantify the effect of these systems on clinical outcomes. Hospitals are "chaotic" systems, and may be impervious to analysis using linear methodology (Kerridge, 2000). In this particular subject area it would appear appropriate to move beyond research that is driven by primary outcome measures. 
RRS are activated predominantly by nurses, and a recurring theme within the literature, is that these systems are under-utilised and under-activated by nurses (Bristow \& Herrick, 2002; Kenward et al., 2004; Hillman et al., 2005). This underutilisation suggests that many opportunities for early interventions for deteriorating ward patients are missed. Patients continue to meet criteria that should activate the efferent limb but, these limbs continue not be activated (Hillman et al., 2005) and although this clinical problem has been identified (Kenward et al., 2004; Hillman et al., 2005;) there is a lack of data explaining the extent of the problem, and why it occurs. The reasons why nurses appear reluctant to activate these systems are therefore worthy of further investigation.

Many of the studies that demonstrate a favourable impact of the RRS have been criticised for their research methodologies. Five studies used case-mix adjustments (Table 1), which is problematic for a number of reasons. Case-mix adjustment does not include differences in patient populations, funding levels, staffing ratios, medical and nursing staff expertise, and "cultural" differences between hospitals (Kerridge 2000). The MERIT (Medical Early Response Intervention and Therapy Study) (Hillman et al., 2005) trial and the study by Bellomo and colleagues (2003) used twelve month implementation period. In an environment as complex and chaotic as an acute care hospital a longer implementation period may be required for the significant cultural change required to assimilate RRS into clinical practice.

\section{Strengths and Limitations}

This review has a number of strengths. It uses the model proposed in the first consensus statement on RRS to explore, analyse and synthesise the empirical data on the RRS. This has enabled a transparent analysis of the contribution of the efferent and afferent limbs of the RRS in optimising the deteriorating ward patients' clinical outcomes.

It would be naïve not to recognise the limitations of this review. First, only papers written in English were included. This inclusion criterion may have excluded some important and relevant studies. Second, the inclusion criteria dictated that only studies 
using data that explored the effects of RRS on in-hospital cardiac arrest, unplanned admission to ICU and death were included. The incorporation of other methodological studies may have highlighted important subjective factors which may hinder or support the introduction of these complex systems.

\section{Relevance to clinical practice}

RRS are in the process of evolution and development within the acute care setting. The ongoing debates regarding RRS effectiveness guarantee RRS a continued presence within the clinical, educational, research and political arena. DeVita et al., (2006) highlight the multidimensional nature of RRS although, previous papers have explored only one aspect of the system. This review explores the importance of the afferent and efferent limb in relation to RRS and, thus ensures a more comprehensive evaluation of RRS. However, what appears to be lacking within the literature is an indepth exploration of all the elements of RRS. Clearly, there is an urgent need for future research to explore all of the factors that make up RRS. Future research into RRS should be robustly planned and implemented to build on current knowledge and understanding of RRS. Qualitative research methods should be used to explore the contextual issues that may affect clinicians' utilisation of RRS. Large, multi-centre RCTs should be conducted, with an international focus, which will determine clinically important outcomes: reduction in in-hospital cardiac arrests, unplanned admission to ICU and reduced morbidity and mortality.

\section{Conclusion}

The care of the critically ill patient increasingly takes place outside the confines of the critical care unit. This significant clinical problem needs to be constructively addressed. RRS provides a rational and logical model that may improve patient care, and at the least is unlikely to make things worse. This review has illustrated two important gaps within the literature. First, 'ramp up' systems have not been subjected to formal evaluation in relation to their impact on MAEs. Second, RRS are under activated and underutilised by practitioners, particularly nurses. There is an urgent need to explore the reasons for this and identify interventions to improve activation of these systems in an effort to promote safe and effective care 


\section{Contributions}

Study design: DM, LA, WC; Data collection and analysis: DM, LA, WC and manuscript preparation: DM,LA,WC.

\section{Conflict of interest.}

No conflict of interest 


\section{References}

Australian, Institute of Health and Welfare. 2008. Australian Health 2008. The Eleventh Biennial Health Report of the Australian, Canberra: Australian Institute of Health and Welfare

Buist M., Bernard S. \& Anderson J. (2003): Epidemiology and prevention of unexpected in-hospital deaths. Surgeon 1, 265-8.

Massey D., Aitken L.M. \& Wendy C. (2008): What factors influence suboptimal ward care in the acutely ill ward patient? Australian Critical Care 21, 127-40.

McGloin H., Adam S.K. \& Singer M. (1999): Unexpected deaths and referrals to intensive care of patients on general wards. Are some cases potentially avoidable? Journal of the Royal College of Physicians London 33, 255-9.

Buist M., Bernard S., Nguyen T.V., Moore G. \& Anderson J. (2004): Association between clinically abnormal observations and subsequent in-hospital mortality: a prospective study. Resuscitation 62, 137-41.

Buist M.D., Jarmolowski E., Burton P.R., Bernard S.A., Waxman B.P. \& Anderson J. (1999): Recognising clinical instability in hospital patients before cardiac arrest or unplanned admission to intensive care. A pilot study in a tertiary-care hospital. Medical Journal of Australia 171, 22-5.

Cretikos M., Parr M., Hillman K., Bishop G., Brown D., Daffurn K., Dinh H., Francis N., Heath T., Hill G., Murphy J., Sanchez D., Santiano N. \& Young L. (2006): Guidelines for the uniform reporting of data for Medical Emergency Teams. Resuscitation 68, 11-25.

Hillman K., Chen J., Cretikos M., Bellomo R., Brown D., Doig G., Finfer S. \& Flabouris A. (2005): Introduction of the medical emergency team (MET) system: a cluster-randomised controlled trial. The Lancet 365, 2091-7.

Bellomo R., Goldsmith D. \& Uchino S. (2004): Prospective controlled trial of effect of medical emergency team on postoperative morbidity and mortality rates. Critical Care Medicine 32, 916 - 921.

Buist M., Moore G., Bernard S., Waxman B., Anderson J. \& Nguyen T. (2002): Effects of a medical emergency team on reduction of incidence of and mortality from unexpected cardiac arrests in hospital: preliminary study. British Medical Journal 324, 387-90.

Cretikos M. \& Hillman K. (2003): The medical emergency team: does it really make a difference? Internal Medical Journal 33, 511-4.

Cretikos M.A., Chen J., Hillman K.M., Bellomo R., Finfer S.R. \& Flabouris A. (2007a): The effectiveness of implementation of the medical emergency team (MET) system and factors associated with use during the MERIT study. Critical Care Resuscation 9, 206-12.

DeVita M.A., Bellomo R., Hillman K., Kellum J., Rotondi A., Teres D., Auerbach A., Chen W., Duncan K., Kenward G., Bell M., Buist M., Chen J., Bion J., Kirby A., Lighthall G., Ovreveit J., Braithwaite R.S., Gosbee J., Milbrandt E., Peberdy M., Savitz L., Young L., Harvey M. \& Galhotra S. (2006): Findings of the first consensus conference on medical emergency teams Critical Care Medicine 34, 2463-2478.

Priestley G., Watson W., Rashidian A., Mozley C., Russell D., Wilson J., Cope J., Hart D., Kay D., Cowley K. \& Pateraki J. (2004): Introducing Critical Care Outreach: a ward-randomised trial of phased introduction in a general hospital. Intensive Care Medicine 30, 1398-404. 
Gao H., McDonnell A., Harrison D.A., Moore T., Adam S., Daly K., Esmonde L., Goldhill D.R., Parry G.J., Rashidian A., Subbe C.P. \& Harvey S. (2007): Systematic review and evaluation of physiological track and trigger warning systems for identifying at-risk patients on the ward. Intensive Care Medicine 33, 667-79.

Esmonde L., McDonnell A., Ball C., Waskett C., Morgan R., Rashidian A., Bray K., Adam S. \& Harvey S. (2006): Investigating the effectiveness of critical care outreach services: a systematic review. Intensive Care Medicine 32, 1713-21.

McGaughey J., Alderdice F., Fowler R., Kapila A., Mayhew A. \& Moutray M. (2007): Outreach and Early Warning Systems (EWS) for the prevention of intensive care admission and death of critically ill adult patients on general hospital wards. Cochrane Database Systematic Review.

Whittemore R. \& Knafl K. (2005): The integrative review: updated methodology. Journal of Advanced Nursing 52, 546-53.

Conn V. \& Rantz M. (2003): Research methods: Managing primary study quality in meta-analyses. Research in Nursing \& Health 26, 322-333.

O'Mathuna D.P. (2000): Evidence-based practice and reviews of therapeutic touch. Journal of Nursing Scholarship 32, 279-85.

Ball C., Kirkby M. \& Williams S. (2003): Effect of the critical care outreach team on patient survival to discharge from hospital and readmission to critical care: non-randomised population based study. British Medical Journal 327, 1014.

Bellomo R., Goldsmith D., Uchino S., Buckmaster J., Hart G.K., Opdam H., Silvester W., Doolan L. \& Gutteridge G. (2003): A prospective before-and-after trial of a medical emergency team. Medical Journal of Australia 179, 283-7.

Robson W.P. (2002): An evaluation of the evidence base related to critical care outreach teams -- 2 years on from Comprehensive Critical Care. Intensive \& Critical Care Nursing 18, 211-8.

Brown S.A., Upchurch S.L. \& Acton G.J. (2003): A Framework for Developing a Coding Scheme for Meta-Analysis. Western Journal of Nursing Research 25, 205-222.

Cuthbertson B.H.M.D.F., Boroujerdi M.P., McKie L.M.B.C., Aucott L.P. \& Prescott G.P. (2007): Can physiological variables and early warning scoring systems allow early recognition of the deteriorating surgical patient? Critical Care Medicine 35, 402-409.

Cretikos M., Chen J., Hillman K., Bellomo R., Finfer S. \& Flabouris A. (2007b): The objective medical emergency team activation criteria: a case-control study. Resuscitation 73, 62-72.

Gardner-Thorpe J., Love N., Wrightson J., Walsh S. \& Keeling N. (2006): The value of Modified Early Warning Score (MEWS) in surgical in-patients: a prospective observational study. Annalsl of the Royal College of Surgery England 88, 571-5.

Subbe C.P., Davies R.G., Williams E., Rutherford P. \& Gemmell L. (2003): Effect of introducing the Modified Early Warning score on clinical outcomes, cardiopulmonary arrests and intensive care utilisation in acute medical admissions. Anaesthesia 58, 797-802.

Morgan R. \& Wright F. (1997): An Early Warning Scoring system for detecting developing critical illness. Clinical Intensive Care 8, 100

McArthur-Rouse F. (2001): Critical care outreach services and early warning scoring systems: a review of the literature. Journal of Advanced Nursing 36, 696-704. 
Cullinane M., Findlay G., Hargraves C. \& Lucas S. (2005) An Acute Problem? NCEPOD 2005 Report HMSO London

NICE (2007) Acutely ill patients in hospital (NHS, ed). National Institute for Health and Clinical Excellence, London

Needleman J., Buerhaus P., Mattke S., Stewart M. \& Zelevinsky K. (2002): Nursestaffing levels and the quality of care in hospitals. New England Journal of Medicine 346, 1715-22.

Stenhouse C., Coates S., Tivey M., Allsop P. \& Parker T. (2000): Prospective evaluation of a modified Early Warning Score to aid earlier detection of patients developing critical illness on a general surgical ward. British Journal of Anaesthesia 84, 663-.

Subbe C.P., Kruger M., Rutherford P. \& Gemmel L. (2001): Validation of a modified Early Warning Score in medical admissions. Quality Journal Medicine 94, 521-6.

Duckitt R.W., Buxton-Thomas R., Walker J., Cheek E., Bewick V., Venn R. \& Forni L.G. (2007): Worthing physiological scoring system: derivation and validation of a physiological early-warning system for medical admissions. An observational, population-based single-centre study. British Journal of Anaesthesia 98, 769-74.

Hodgetts T.J., Kenward G., Vlachonikolis I.G., Payne S. \& Castle N. (2002): The identification of risk factors for cardiac arrest and formulation of activation criteria to alert a medical emergency team. Resuscitation 54, 125-31.

Coombs M. \& Dillon A. (2002): Crossing boundaries, re-defining care: the role of the critical care outreach team. Journal of Clinical Nursing 11, 387-93.

Lee A., Bishop G., Hillman K.M. \& Daffurn K. (1995): The Medical Emergency Team. Anaesthesia Intensive Care 23, 183-6.

Ranji S.R., Auerbach A.D., Hurd C.J., O'Rourke K. \& Shojania K.G. (2007): Effects of rapid response systems on clinical outcomes: systematic review and metaanalysis. Journal of Hospital Medicine 2, 422-32.

Winters B.D., Pham J.C., Hunt E.A., Guallar E., Berenholtz S. \& Pronovost P.J. (2007): Rapid response systems: a systematic review. Critical Care Medicine 35, 1238-43.

Dacey M.J., Mirza E.R., Wilcox V., Doherty M., Mello J., Boyer A., Gates J., Brothers T. \& Baute R. (2007): The effect of a rapid response team on major clinical outcome measures in a community hospital. Critical Care Medicine 35, 2076-82.

DeVita M.A., Braithwaite R.S., Mahidhara R., Stuart S., Foraida M. \& Simmons R.L. (2004): Use of medical emergency team responses to reduce hospital cardiopulmonary arrests. Quality \& Safety in Health Care 13, 251-254.

Jones D., Bellomo R., Bates S., Warrillow S., Goldsmith D., Hart G., Opdam H. \& Gutteridge G. (2005): Long term effect of a medical emergency team on cardiac arrests in a teaching hospital. Critical Care Medicine 9, R808-15.

Bristow P.J., Hillman K.M., Chey T., Daffurn K., Jacques T.C., Norman S.L., Bishop G.F. \& Simmons E.G. (2000): Rates of in-hospital arrests, deaths and intensive care admissions: the effect of a medical emergency team. Medical Journal of Australia 173, 236-40.

Kenward G., Castle N., Hodgetts T. \& Shaikh L. (2004): Evaluation of a medical emergency team one year after implementation. Resuscitation 61, 257-63. 
Cretikos M.A., Bellomo R., Hillman K., Chen J., Finfer S. \& Flabouris A. (2008): Respiratory rate: the neglected vital sign. Medical Journal of Australia 188, 657-9.

Fieselmann J.F., Hendryx M.S., Helms C.M. \& Wakefield D.S. (1993): Respiratory rate predicts cardiopulmonary arrest for internal medicine inpatients. Journal of General Internal Medicine 8, 354-60.

Kellett J. \& Deane B. (2006): The Simple Clinical Score predicts mortality for 30 days after admission to an acute medical unit. Quality Journal Medicine 99, 771-781.

Goldhill D.R. \& McNarry A.F. (2004): Physiological abnormalities in early warning scores are related to mortality in adult inpatients. British Journal of Anaesthesia 92, 882-4.

Kerridge R. (2000): The medical emergency team: no evidence to justify not implementing change'. Medical Journal of Australia 173, 228-229.

Bristow D.P. \& Herrick C.A. (2002): Emergency department case management: the dyad team of nurse case manager and social worker improve discharge planning and patient and staff satisfaction while decreasing inappropriate admissions and costs: a literature review. Lippincotts Case Management7, 243-51. 